\title{
Evidencia del síndrome neuropático en un estudio neurofisiológico e inmunohistoquímico de las fibras nerviosas en pacientes con síndrome de boca ardiente
}

\author{
Lauritano D*, Calzavara D**, Papagna R*, Baldoni $M^{* * *}$, Bascones $A^{* * * * *}$
}

\section{RESUMEN}

Introducción: El síndrome de la boca ardiente (SBA) es una enfermedad de frecuente aparición, caracterizada para un dolor quemante y ardiente en la lengua y en la cavidad oral, en ausencia de alteraciones estructurales macroscópicas de las mucosas. La etiopatogénesis del SBA no es todavía conocida y por lo tanto no presenta un esquema diagnóstico claro y bien definido.

Material y métodos: Para evaluar la hipótesis que contempla una alteración de las fibras nerviosas periféricas de pequeño calibre, asociada o no con una alteración trófica de las mucosas, hemos seleccionados 25 pacientes (7 varones y 18 mujeres, con una edad comprendida entre 36 y 75 años, una media de 54 años) afectados por SBA, que llegaron a nuestra clínica para una serie de exámenes que suelen realizarse de forma rutinaria en las síndromes de dolor neurológico. En todos los pacientes se ha realizado un cuidadoso examen objetivo y una ortopantomografía, con la finalidad de excluir alteraciones de las mucosas y enfermedades odontológicas. En todos los pacientes se han realizado: exámenes hematoquímicos, exámenes objetivos neurológicos, teletermografía de la lengua y de la cara, examen cuantitativo de la sensibilidad térmica de la lengua, de las manos y de la parte dorsal del pie. Los resultados del examen cuantitativo de la sensibilidad térmica de la lengua y de la muñeca se confrontaban con una población control homogénea. En una población de 10 pacientes ( 3 varones y 7 mujeres; de una edad comprendida entre 34 y 53 años, una media de 49 años) se ha realizado un biopsia de la parte dorsal de la lengua, la biopsias se han analizado con microscopía óptica e inmunofluresencia tras un tratamiento con anticuerpos activos para proteínas neuronales citoplasmáticas (protein gene product 9.5).

Resultados: Los datos obtenidos evidenciaban la presencia de una polineuropatía subclínica en el $50 \%$ de los pacientes. Especialmente se apreciaban signos de baja funcionalidad de las fibras nerviosas de pequeño calibre de la lengua en casi la mitad de los pacientes examinados. El examen histológico de la mucosa lingual evidenciaba una atrofia discreta en el $70 \%$ de los pacientes, mientras que la observación de las fibras nerviosas de pequeño calibre por medio de la inmunofluoresencia hacía suponer la presencia de alteraciones relacionadas con una polineuropatía de tipo periférico.

Palabras Clave: Boca ardiente. Xerostomía. Mucosas orales.

\section{SUMMARY}

Aims. Burning pain is considered characteristic of chronic neuropathic pain condition in general, and some recent data seem to suggest peripheral or central nervous system involvement as possible underlying factor in BMS. This 
study was designed to determinate if burning mouth symptoms could be originated from a peripheal neuropathy of small diameter nerve fibres.

Material and methods. The material of the study consisted of 25 patients (18 female, 7 male, 30-75 years; means 54 years). In all patients there were not: oral muco membranes lesions, oral muco membranes diseases, oral correlated diseases, oral dysfunctions, dental and periodontal diseases. Besides there were: no evidence of central nervous system pathology, no evidence of peripheral nervous system pathology, no presence of organic body disfunctions. All patients didn't show significant alterations of blood investigations. All patients were undermitted to our protocol including: oral and facial clinical examinations, neurological exam, blood investigations, Mc Gill Pain Questionnaire, VAS (visual analogical scale), biopsy of tongue mucosa with hystological and immunofluorescence exams, quantitative somatosensory thermotest and teletermography examination of tongue mucosa.

Results. These examinations have showed subclinical polineurophaty in $50 \%$ of patients. In particular were observed a loss of function in small diameter nervous fibres in about $50 \%$ of patients. Hystological exam of mucosal tongue revealed a moderate atrophy and alterations of structures.

Conclusion. Etiology, pathogenetic processes, clinical and diagnostic approaches, therapeutical resolutions and researches of burning mouth syndrome are not clear yet. The local alterations of small diameter sensitive nerve fibres could cause an increase of oral burning, without muco membranes pathologies. These local lesions could justify the bms clinical symptomatology. The hystological alteration of small diameter sensitive nerve fibres could involve meaningful alterations of thermal reactivity of oral and body surfaces. These clinical reactivities are evident in chronic peripheal neuropathy (diabetes). These conclusions and our study results are still provisory. It will be necessary to estend and to increase our results, comparing our research with other bms etio-pathogenetic hypotesis.

Key Words: Burning mouth. Xerostomia. Oral mucosa.

* Odontólogo Colaborador de la Clínica Odontoiatrica, Università degli Studi di Milano-Bicocca.

** Odontólogo y Master Periodoncia Universidad Complutense.

*** Profesor Ordinario, Facoltá di Medicina e Chirurgia Università degli Studi di Milano-Bicocca.

***** Catedrático de Medicina Bucal y Periodoncia. Universidad Complutense de Madrid.

Lauritano D, Calzavara D, Papagna R, Baldoni M, Bascones A. Evidencia del síndrome neuropático en un estudio neurofisiológico e inmunohistoquímico de las fibras nerviosas en pacientes con síndrome de boca ardiente. Av. Odontoestomatol 2003; 19-2: 81-94.

\section{INTRODUCCIÓN}

El Síndrome de la Boca Ardiente (SBA) está caracterizado para una sintomatología dolorosa de naturaleza idiopática, con un característico dolor quemante a lo largo de la lengua y de la mucosa oral (1). La Sociedad Internacional del Estudio del Dolor (I.A.S.P) define la SBA como"una sensación de dolor quemante que comprende la lengua $\mathrm{y} / \mathrm{u}$ otras mucosas de la boca en ausencia de enfermedades locales". El síntoma patognomónico del SBA es una sensación de dolor quemante, a veces muy intenso (23). Generalmente están centrados en la punta de la lengua y los labios, casi siempre bilateral, en menor medida están afectados el paladar y otras localizaciones en la cavidad oral. La mayoría de los pacientes refiere quemazón al mismo tiempo en varias zonas de la cavidad oral, también la zona que suele ser más dolorosa es la punta de la lengua (4-9). Los adjetivos de McGill "pain questionnaire" que se utilizan en la mayoría de los pacientes afectados por SBA con la finalidad de describir la calidad de su molestia, son: quemante, debilitante, aburrido (10). La intensidad del dolor es parecida a la odontálgica, si bien de calidad distinta. Empleando una escala visio-analógica del dolor, con valores desde 0 hasta 10, que corresponde a la máxima intensidad del dolor probado en el pasado, de media la intensidad 
Lauritano D, Calzavara D, Papagna R, Baldoni M, Bascones A Evidencia del síndrome neuropático en un estudio neurofisiológico e inmunohistoquímico de las fibras nerviosas en pacientes con síndrome de boca ardiente

del dolor del SBA se valoraba como 8 (11). El dolor frecuentemente es exacerbado por situaciones de estrés sea psicológico como físico, cansancio o problemas sociales (12). Algunos pacientes presentan un empeoramiento del dolor durante las comidas, mientras que otros contrariamente presentan un alivio durante la masticación. El calor atmosférico suele empeorar el dolor. Eventos que distraen al paciente y una temperatura atmosférica fría pueden asociarse con una disminución del dolor (13). Se ha propuesto una clasificación del SBA sobre la base de la intensidad de la sensación de quemazón: SBA de grado leve, moderado y grave. Una segunda clasificación diferencia tres tipologías de SBA sobre la base de la variación diaria de los síntomas. Los pacientes con SBA de tipo 1 no presentan síntomas al despertarse; luego la molestia aparece por la mañana, aumenta de forma progresiva a lo largo del día hasta llegar al máximo de la intensidad en las primeras horas de la noche. Los pacientes que presentan el SBA de tipo 2 refieren una sensación de ardor que se queda inalterada a lo largo de todo el día. Típicamente los pacientes afectados por el SBA de tipo 1 y 2 no presentan una sintomatología ardiente durante las horas de la noche. Finalmente el SBA de tipo 3 está caracterizado para una alternancia de periodos de ardor y otros de ausencia del síntoma (14). En más de dos tercios de los pacientes con el SBA se han apreciado alteraciones de gusto, sobre todo por una constante percepción alterada de los sabores. Esta disgeusia se reduce tras un enjuague de la boca con agua (15). Frecuentemente los sujetos con SBA refieren molestias del olfato, xerostomía en casi el $40 \%$ de los casos, que aparece más a menudo tras el principio del ardor oral y dificultades en la deglución en casi el 50\% de la misma población (16).

Frecuentemente además de ardor se asocian al SBA otras molestias inespecíficas como la cefalea, referida en casi el $30 \%$ de los sujetos, dolor a la cervical, colon irritable, dismenorrea, picor, insomnio nocturno y/o hipersomnia durante el día, disminución de la líbido, alteración del humor con irritabilidad, depresión del humor y disminución de la socialización (17).

Se aprecia que los pacientes afectados por SBA presentan a menudo una historia de infancia complica$\mathrm{da}$, problemas familiares, conflictos de pareja, pro- blemas en el trabajo y finalmente problemas económicos. Alteraciones del perfil psicológico, evaluados con exámenes psicométricos, se han visto en un paracentaje elevado en los pacientes afectados por SBA. Especialmente se ha evidenciado un aumento de las alteraciones de ansia y de depresión, pero también una tendencia a la somatización (18-27).

Las personas afectadas por SBA presentan con una mayor frecuencia que la población normal otros síndromes dolorosos; en estos casos el dolor referido es muchas veces mayor de lo referido usualmente para una patología dolorosa unitaria (28-29).

El SBA es una molestia de tipo crónico, pero más de la mitad de las veces los pacientes presentan una remisión espontánea, completa o parcial del ardor tras 6-7 años (14).

El examen objetivo de la cavidad oral no presenta particulares anormalidades, como signos de inflamación o de atrofia de la mucosa; esta condición es necesaria para establecer el diagnóstico del SBA. Se ha apreciado frecuentemente la presencia de alguna alteración en la percepción de los sabores, sin embargo la capacidad de distinción de diferentes sabores se mantiene (13). No se ha apreciado una diferencia estadísticamente significativa entre pacientes y la población control con respecto a la discriminación de un doble estimulo mecánico y de la estereognósia de la lengua. La xerostomía esta frecuentemente referida por parte de los pacientes afectados por SBA, sin embargo no se ha revelado una disminución de la secreción salivar tanto en ausencia de estimulación como tras la estimulación de las glándulas salivares. Pero se ha revelado en algunos pacientes una diferente composición de la saliva (30-33).

Los exámenes hematoquímicos generalmente se presentan alterados en un porcentaje limitado de pacientes. Algún paciente afectado por SBA, más o menos el 3\%, presenta niveles elevados de glicemia; el $39 \%$ de los pacientes con SBA presentan una intolerancia a la glucosa, revelada a través de pruebas de carga de glucosa, aunque otros investigadores no han encontrado datos significativos para seguir investigando por este camino. Se ha planteado también la hipótesis que relaciona el SBA de los diabéti- 
cos con el tipo de insulina empleada, pero otros estudios rechazan esta hipótesis (34).

El SBA es una molestia de tipo autónomo, que se diferencia de otras enfermedades caracterizadas por ardor de la cavidad oral pero asociadas a signos de mucositas o alteraciones de la mucosa oral (35). Por el contrario la definición del SBA tiene que ser restringida a los casos donde no es posible evidenciar a nivel macroscópico signos de patología de la mucosa oral, de causa infecciosa, alérgica, química o mecánica. Desgraciadamente en la literatura se ha confundido frecuentemente el dolor ardiente con el SBA verdadero y por lo tanto los casos que se refieren a esta patología no siempre son atendibles, incluyendo casos de ardor de causa infecciosa, hipersensibilidad y otras causas de patología oral (36-37).

El diagnóstico diferencial entre SBA y otras patologías hay que ponerlo sobre la base del examen clínico de la mucosa. El examen citológico y fúngico de la mucosa pueden siempre contribuir a la identificación de infecciones fúngicas o bacterianas responsables de la sintomatología ardiente.

\section{UNA NUEVA HIPÓTESIS ETIOPATOGENÉTICA}

Distintos aspectos sintomatológicos y epidemiológicos del SBA sugieren que la patogénesis de esta enfermedad puede ser debida a una alteración neurológica periférica. La característica ardiente del dolor, agravada por los estímulos calientes y aliviada por el frío, junta las manifestaciones del SBA a las neuropatías periféricas dolorosas, que se pueden apreciar en la diabetes o en los procesos infecciosos del hígado. Además el SBA se manifiesta más frecuentemente en las personas que ya presentaban factores de riesgo por una polineuropatía. Ha sido por lo tanto puesta una señal sobre la frecuente asociación con la diabetes (9), causa principal de polineuropatías en nuestro país o con las hipovitaminosis. Los esfuerzos de los investigadores han estado dirigidos a investigar la relación entre estas patologías carenciales o dismetabólicas y eventuales alteraciones de la mucosa, olvidando que estos desequilibrios podrían también ser causa de polineuropatías
(38-40). Nuestro estudio se basa sobre la hipótesis de que pueda existir por lo menos en una sub-población de sujetos afectados por SBA, una polineuropatía que se expresa en la cavidad oral con un dolor ardiente. Hemos por lo tanto presentado la hipótesis de que, la cavidad oral puede ser una localización principalmente interesada para un dolor ardiente en el curso de una polineuropatía de pequeñas fibras nerviosas. Los fenómenos descritos asociados a las lesiones de las fibras nerviosas de pequeño calibre son difícilmente detectables en la práctica clínica. Sin embargo la presencia de un déficit de la sensibilidad al calor y al frío es un indicador directo de lesiones de estas fibras y por lo tanto se asocia a los fenómenos positivos de "irritación nerviosa" descritos.

Con el presente trabajo nos hemos planteado investigar la presencia de los signos de sufrimiento de las fibras nerviosas sensitivas y especialmente de las molestias sensitivas de la cavidad oral. Hemos por lo tanto organizado un estudio compuesto de una investigación de tipo neurofisiológica inmunohistoquímica, con la finalidad de examinar una posible asociación entre el SBA y las patologías neurológicas periféricas o causas conocidas de polineuropatía. Nuestro protocolo de trabajo es normalmente aplicado en los estudios de las polineuropatías dolorosas con localización a nivel de la cavidad oral.

\section{CAsos cLínICOS}

En el departamento de Medicina y Patología Oral de la Clínica Odontológica de la Università degli Studi di Milano-Bicocca han pasado bajo nuestra observación 25 pacientes con sintomatología ardiente a nivel de la cavidad oral. De estos 18 eran mujeres, 7 varones con una edad media de 54 años. El sujeto más joven tenia 36 años y el más viejo 75 años. Estos pacientes se han incluido en nuestro protocolo de investigación porque cumplían los siguientes criterios de inclusión:

1) Estar afectados por el SBA diagnosticado clínicamente según los parámetros de la Sociedad Internacional para el Estudio del Dolor.

2) Referir ardor oral desde por lo menos tres meses. 
3) Tener una edad entre los 25-75 años. Hemos observado también pacientes de una edad superior a los 75 años, sin embargo hemos preferido excluirlos de esta población, para evitar una disformidad de los datos. Es bien conocido que, el umbral de percepción de los estímulos térmicos presentan mínimas variaciones intra-individuales a lo largo de la vida con la excepción de la tercera edad, donde se aprecia tras los 80 años un significativo incremento del umbral de percepción de los estímulos calientes en las extremidades inferiores (41).

4) No estar afectado por patologías del sistema nervioso central y no estar en tratamiento con psicofármacos tales que creen sedación y retraso en la respuesta a los estímulos proporcionados. Esta prerrogativa era indispensable para una correcta ejecución del examen del umbral de la sensibilidad a los estímulos térmicos.

5) Adhesión completa y voluntaria a todas las fases del estudio.

\section{MATERIAL Y MÉTODOS}

En todos los pacientes se han realizados:

- Examen objetivo local de la cavidad oral: en los pacientes una vez realizado el examen de las mucosas orales éstas presentaban un aspecto normal, de color rosa, normalmente mojadas, regularmente humidificadas por un constante flujo de saliva; no se apreciaban signos de patologías orales. El examen de las estructuras dento-periodontales evidenciaba en general una situación aceptable, sea por los aspectos odontológicos sea por la higiene oral. 15 pacientes eran portadores de prótesis total.

- Toma de datos anamnésicos y clínicos. Ha sido recopilada una ficha clínica que contemplaba la anamnesis de patología anterior, patología próxima, sea sistémica o específica, las terapias en curso y una descripción del examen objetivo odontoestomatológico. En la ficha clínica se apuntaban también el presunto diagnóstico, el programa tera- péutico, las visitas de control y el diagnóstico de certeza.

Se hacía recopilar al paciente un cuestionario que contemplaba simples preguntas con referencia a los hábitos nocivos, tabaco, consumo de alcohol, tipo de alimentación, la frecuencia de las visitas de control odontoestomatológicas.

Todos los datos así recopilados eran sucesivamente codificados en un programa informático para su grabación y un posible uso estadístico de los mismos.

En cada una de las fichas se colocaba el material iconográfico con diapositivas realizadas en la primera visita.

Para cada paciente se contemplaba una serie de exámenes de laboratorio, que eran realizados en el mismo laboratorio de análisis. Estos exámenes de laboratorio contemplaban: el hematocrito con la fórmula leucocitaria, la glucemia, la azotemia, la VES, la sideremia, la feritina, el índice de saturación de la transferina, los valores de las transaminasas (GOT, GPT, las gamaGT), marcadores de la hepatitis B y C, la vitamina b12, los folatos, las IG séricas, los valores de C3, C4 y las fracciones del complemento. En cada paciente se prescribía una radiografía panorámica para detectar la presencia de posibles patologías dentarias.

- Examen objetivo neurológico.

- Examen cuantitativo de las sensibilidad térmica de la lengua, de la eminencia tenar de la mano izquierda y al dorso del pie derecho; este examen se realizaba además también en una población control de la misma edad para la comparación estática de los datos.

Para la evaluación cuantitativa de la sensibilidad térmica de la mucosa lingual se utilizaba un instrumento estimulante de la superficie de $12,5 \mathrm{~cm}^{2}$, compuesto de elementos de Peltier, dotados de la característica de refrigerarse o calentarse tras el paso de corriente eléctrica, y por un termómetro que revelaba la temperatura de la interfase mucosa-estimulador. El estimulador estaba conectado a un dispositivo Medoc TSA $2001^{\oplus}$, que regula la temperatura gra- 
bando los valores. Ha sido evaluado el umbral de percepción de los estímulos clientes, fríos, dolorosos calientes y dolorosos fríos, empleando un esquema "a niveles" de suministración de los estímulos. El estimulador se coloca en contacto con la mucosa lingual del sujeto a examinar; empezando desde una temperatura basal de $32^{\circ} \mathrm{C}$ la superficie del estimulador aumenta o disminuye progresivamente de temperatura hasta que el sujeto señala percibir el estimulo pulsando una tecla. Se calcula por lo tanto la media de los umbrales de percepción de más estímulos, una vez establecido el umbral de percepción para cada modalidad sensorial (caliente, frío, dolor para calor y para frío) (42-43). Los valores obtenidos se comparan con una población control de igual edad. Se calcula además la diferencia del umbral de percepción de los estímulos térmicos entre el dorso de la muñeca y del pie; un valor elevado con referencia a la población control es indicativo de sufrimiento de las fibras nerviosas distales de pequeño calibre (41). De forma análoga se evalúa la diferencia entre los umbrales perceptivos para los estímulos térmicos de la lengua y de la eminencia tenar. Se busca una disminución del umbral de percepción de los estímulos térmicos dolorosos en la lengua (hiperalgesia); una disminución de este umbral con respecto a la población control o con respecto a las otras localizaciones corpóreas es indicativa del síndrome de sensibilidad receptor como la que se aprecia en el curso de las polineuropatías dolorosas (síndrome $\mathrm{ABC})(43)$.

- Teletermografías de la lengua y de la región de le mejilla y de le frente (44).

Se detectaba la temperatura cutánea de la cara y de la mucosa de la lengua con un aparato a rayos infrarrojos $\mathrm{Jeol}^{\circledast}$. La temperatura de la mucosa lingual se comparaba con una población control y con las temperaturas cutáneas de la cara. Un aumento de la temperatura es indicativo de una pérdida de fibras nerviosas de pequeño calibre con acción vaso-dilatadora. Este cuadro se aprecia también en el curso de la liberación de los mediadores de la inflamación desde los terminales sensitivos de las fibras nerviosas de pequeño calibre sensibilizadas (síndrome $A B C$ ) (38).

- En 10 pacientes se realizó la biopsia de la mucosa lingual con la finalidad de realizar una investigación de la inervación de la mucosa lingual con observación a la microscopía óptica y con inmunofluresencia.

Las biopsias se realizaban a nivel de la mucosa del dorso lingual en correspondencia con el borde lingual; eran empleados los punch-biopsy de forma circular del diámetro de $6 \mathrm{~mm}$. Estos instrumentos permitían obtener márgenes de cortes extremamente precisos con un consecuente menor trauma para la zona de la mucosa biopsiada y menos molestias para el paciente. Las lesiones se suturaban con aguas atraumáticas de seda. Las biopsias eran posicionadas y orientadas correctamente sobre un papel bibula, mojados con una solución fisiológica y colocados en contenedores herméticos de laboratorio. El fijador empleado era la formalina tamponada al $10 \%$. Las biopsias así prelavadas se repartían a su vez en dós fragmentos más pequeños de iguales dimensiones. Una biopsia se enviaba al laboratorio de anatomía patológica donde los exámenes histológicos se realizaban con coloraciones estándar en hematoxilina y eosina, mientras la otra se analizaba en el laboratorio de neurofisiología de reparto de neurología del Hospital San Raffaele de Milán, donde se reconstruía la inervación de la mucosa lingual por medio de la observación con inmunofluoresencia. El segundo fragmento de mucosa era tratado con anticuerpos directos para las proteínas neuronales citoplasmáticas (proteínas del gen product 9.5) (45-46). Este marcador permite una nueva técnica para estudiar la identificación y la cuantificación de la distribución de las fibras nerviosas terminales periféricas de la mucosa oral, y permite por lo tanto una comparación entre fibras nerviosas de la mucosa de los sujetos "sanos" y de los sujetos afectados por SBA.

- Evaluación de la intensidad del dolor con un análogo visual de Scott-Huskinsson. La escala visioanalógica es el instrumento que se ha utilizado preferentemente en la práctica clínica en la evaluación de los parámetros unitarios relativos al dolor crónico.

Estos instrumentos de evaluación de la intensidad y de la calidad del dolor y de sus complicaciones asociadas se suministran al paciente en la primera visita. 


\section{RESULTADOS: MCGILL PAIN QUESTIONAIRE e VAS}

El McGill Pain Questionaire (tab.1) ha evidenciado una puntación elevada del total (suma de todos los descriptores) de todos los descriptores, datos confirmado para el valor medio de 6 puntos revelados en la evaluación del dolor por medio de una escala visio-analógica (VAS). Además es evidente la gran diferencia entre la alta media del total de los descriptores relativos a la esfera sensorial en comparación a la baja media de los revelados para la esfera afectiva. Este es un resultado que confirma los datos obtenidos en un estudio anterior (47) disminuyendo la relevancia de la eventual componente psicosomática, frecuentemente mencionada en literatura, en la patogénesis de la enfermedad.

\section{TABLA 1.- CUESTIONARIO DE MCGILL PAIN: PUINTUACIONES RELATIVAS DEL TOTAL DE LOS INDICADORES}

\begin{tabular}{|lr|}
\hline $\begin{array}{l}\text { Puntaciones al los totales de los indicadores } \\
\text { (preguntas 1-20) }\end{array}$ & 29,7 \\
\hline $\begin{array}{l}\text { Indicadores sensoriales: } \\
\text { (preguntas 1-10) }\end{array}$ & 16,2 \\
\hline $\begin{array}{l}\text { Indicadores afectivos: } \\
\text { (preguntas 11-15) }\end{array}$ & 3,9 \\
\hline $\begin{array}{l}\text { Indicadores de evaluación: } \\
\text { (pregunta 16) }\end{array}$ & 2,9 \\
\hline $\begin{array}{l}\text { Indicadores heterogéneos: } \\
\text { (preguntas 17-20) }\end{array}$ & 6,7 \\
\hline
\end{tabular}

\section{RESULTADOS: EXAMEN TELERMOGRÁFICO}

El examen telermográfico no ha evidenciado unas significativas diferencias entre la temperatura de la lengua y la de la mejilla. Este test se había realizado en el intento de documentar un aumento de la temperatura local como signo característico de la inflamación neurológica. Hay que considerar todavía que existen importantes dificultades técnicas que obstaculizan una revelación real de la temperatura de la lengua. Por ejemplo, cuando la lengua está expuesta fuera de la cavidad oral se refrigera por la evapora- ción de la película salivar en contacto de la temperatura del ambiente externo. En segundo lugar existen las diferencias significativas entre la temperatura elevada sobre la punta de la mucosa lingual en relación a la base; este factor está asociado a las dificultades de ciertos pacientes en la protrusión de la lengua y a la escasa maniobrabilidad del sistema de la revelación teletermográfica, hacen que los datos obtenidos sean de difícil interpretación.

\section{RESULTADOS: EXAMEN CUANTITATIVO DE LAS SENSIBILIDADES}

Los datos que se han obtenido en el examen cuantitativo de las sensibilidades térmicas, realizado sobre los 25 pacientes y sobre 22 de control, se han representado en las tablas 2 y 3 que contemplan:

a) Umbral de percepción al calor y al dolor caliente de la lengua (tab. 2).

TABLA 2.- VALORES REVELADOS CON EL TEST DE LA SENSIBILIDAD DE LA LENGUA: UMBRAL DE LA PERCEPCIÓN DEL CALOR, DEL FRÍO DEL DOLOR AL CALOR Y AL FRÍO

\begin{tabular}{|lcccc|}
\hline & \multicolumn{2}{c}{ Media } & DS & \multicolumn{2}{c|}{ Control } \\
\hline Calor & 36,8 & 4,2 & 34,8 & 1,9 \\
\hline Frío & 26,9 & 3,9 & 28,6 & 2,3 \\
\hline Dolor calor & 39,8 & 3,9 & 33,7 & 2,3 \\
\hline Dolor frío & 25,3 & 3,1 & 27,2 & 1,4 \\
\hline
\end{tabular}

Esta presentaba un valor medio de $36,8^{\circ} \mathrm{C}$ con dev. est. de $+/-4,2$ y no significativamente diferentes con respecto a la población control que ha evidenciado un valor medio de $34,8^{\circ} \mathrm{C}$ con dev. Est de 1,9 . Los valores del dolor caliente presentaban una media de $39,8^{\circ} \mathrm{C}$, con dev. Est de 3.9 en los pacientes con SBA, mientras una media de $33.7^{\circ} \mathrm{C}$ con dev. Est de 2.3 en el grupo control.

b) Umbral de percepción para el frío y para el dolor por frío de la lengua (tab. 2). 
Como la anterior no evidenciaba valores dignos de ser mencionados; el valor medio de los pacientes es de $-26,95^{\circ} \mathrm{C}$ con dev. Est de 3,9 mientras que los sujetos control evidenciaban un valor medio de +1 $28,65^{\circ} \mathrm{C}$ con dev.est de 2,3. Dolor para el frío: media de $25.3^{\circ} \mathrm{C}$ con dev.est de 3.1 en el grupo SBA, media de $27.2^{\circ} \mathrm{C}$ y dev. Est 1.4 en el grupo control.

c) Diferencia entre el umbral de percepción del calor de la lengua, y aquella del calor de la mano (tab. 3).

\begin{tabular}{|lcccc|}
\hline \multicolumn{4}{|c|}{ TABLA 3.- DIFERENCIAS ENTRE EL UMBRAL } \\
DE PERCEPCIÓN DEL CALOR, FRÍO, DOLOR \\
Y DEL ESTÍMULO CALOR Y FRÍO DE LA \\
LENGUA Y DE LA MANO \\
\hline \multicolumn{4}{|c}{ MBA } & \multicolumn{3}{c|}{ Control } \\
\hline Media & DS & Media & DS \\
\hline Calor & 3,28 & 3,88 & 0,76 & 15,5 \\
\hline Frío & 3,2 & 3,8 & 1,3 & 1,9 \\
\hline Dolor calor & 0,4 & 3,8 & 1,5 & 5,2 \\
\hline
\end{tabular}

Los resultados más significativos del estudio son aquellos relativos a este umbral con un valor medio de $3,28^{\circ} \mathrm{C}$ con una dev.est de $+/-3,88$ para los pacientes, mientras evidenciaba un valor medio de $0,76^{\circ} \mathrm{C}$ con dev.est $+/-15.5$ para los sujetos control. Por lo tanto el umbral de percepción del calor de la lengua era ligeramente superior $\left(3,38^{\circ} \mathrm{C}\right)$ en comparación con los de las manos en los pacientes con SBA; mientras resultaba ser solo ligeramente superior $\left(0,7^{\circ} \mathrm{C}\right)$ en los sujetos control. Hemos además revelado una diferencia superior a los $3^{\circ} \mathrm{C}$ entre el umbral de la lengua y el de la mano en el $40 \%$ de los pacientes. Hemos obtenido resultados con significación estadística: con el Test $\mathrm{T}$ de Student relativo a los valores diferenciales entre los umbrales de percepción al calor de la lengua y de la mano se revelaba un valor de 0,0039. Mientras con la Transformación de Fisher, para estos mismos datos se ha obtenido un valor de 0,03 . Estos evidencian una diversidad entre las dos poblaciones estudiadas, pacientes afectos por SBA y sujetos controles. El siguiente gráfico muestra el umbral de sensibilidad al calor de la lengua, en relación con el de la mano, de todos los pacientes y de los sujetos controles. d) Diferencia entre el umbral de percepción al frío de la lengua y de la mano (tab. 3).

El valor medio detectado en los pacientes es de $-3,2^{\circ}$ C con dev.est de +/- 3,8 mientras los sujetos controles evidenciaban un valor de $-1,3^{\circ} \mathrm{C}$ con dev.est de $+/-1,9$. No ha sido revelado nada significativo.

e) Diferencia entre el umbral de percepción del dolor para estímulo caliente de la lengua y de la mano (tab. 3).

El valor medio revelado en los pacientes es de $0,4^{\circ} \mathrm{C}$ con dev. Est de $+/-3,8$, el de los sujetos control de $1,5^{\circ} \mathrm{C}$ con dev. Est de $+/-5,2$. No se ha revelado nada significativo.

f) Diferencia entre el umbral de percepción del dolor para estimulo frío de la lengua y de la mano.

El valor medio de los pacientes es de $-0,003^{\circ} \mathrm{C}$ con dev.est de 23,5; No ha sido revelado nada significativo.

g) Diferencia entre el umbral de percepción del calor de la lengua y del pie.

El valor medio de los pacientes era de $-3,7^{\circ} \mathrm{C}$ con dev.est de 3,8; El dato no ha sido comparado con los sujetos del segundo grupo.

h) Diferencia entre el umbral de percepción al frío de la mano y del pie.

El valor medio de los pacientes es de $3,01^{\circ} \mathrm{C}$ con dev.est. de $+/-5,1$. Este dato, como lo relativo al punto anterior $(\mathrm{g})$ evidencia una interesante disminución del la sensibilidad de los pies.

i) Diferencia entre el umbral de percepción para el dolor para estimulo de calor de la mano y la del pie.

El valor medio de los pacientes es resultado $-0,12^{\circ}$ C con dev. Est de $+/-3,3$. El dato no ha sido comparado con los sujetos del grupo control; no se ha revelado nada significativo.

l) Diferencia entre el umbral de percepción dolorosa para estimulo frío de la mano y la del pie. 
El valor medio de los pacientes es resultado de $0,5^{\circ}$ $\mathrm{C}$ con dev. Est de +/- 2,4. El dato no se ha comparado con lo de los sujetos controles. No se ha revelado nada significativo. Las pruebas que se han realizado en el pie no se han referido a los sujetos control.

\section{RESULTADOS: HISTOLOGÍA Y INMUNOHISTOQUÍMICA}

El aspecto morfológico con la coloración con hematoxilina / eosina no ha evidenciado en la mucosa oral de los pacientes afectos por SBA particulares diferencias con respecto a los controles (Fig. 1).

En los cortes histológico de los sujetos con SBA, tras el tratamiento con inmunofluoresencia por medio del PGP 9.5, se han apreciado varias fibras nerviosas inmunoreactivas distribuidas superficialmente en la lámina propia, a lo largo de las fascias que forman una red subepitelial dispuesta paralelamente a la superficie. Desde este plexo se originan fibras más sutiles semejantes a pequeñas "venas varicosas" que se dirigen perpendicularmente a la superficie de las papilas, frecuentemente ramificándose, otras veces terminando cerca del epitelio de unión. fascias de fibras nerviosas mas finas se han apreciado en la lámina propia profunda, frecuentemente con localización paralela a los vasos sanguíneos. Ha sido además evidenciada una red de fibras unitarias nerviosas terminales pegadas a las paredes de los vasos sanguíneos (fig.2).

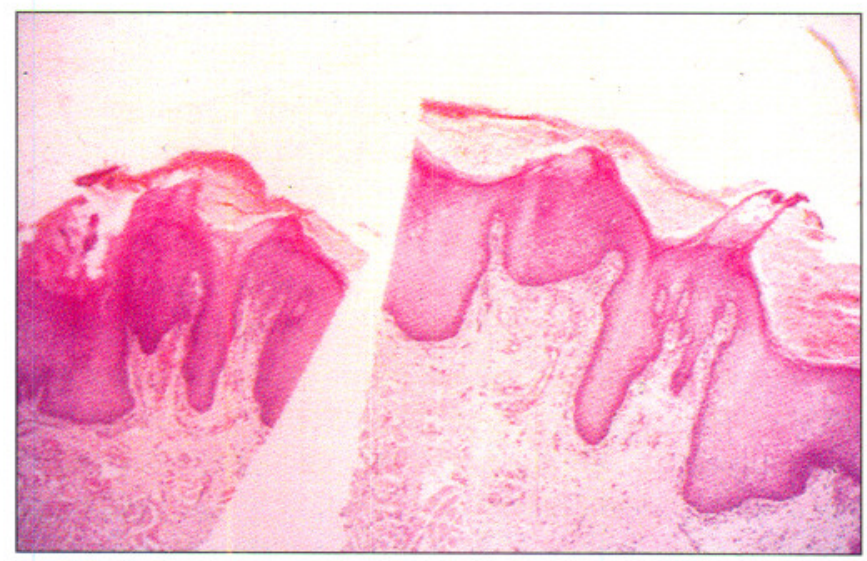

Fig. 1. Examen histológico con coloración hematoxilina-eosina de la mucosa oral de un sujeto con SBA (x40).
Estas observaciones no permiten concluir que las fibras nerviosas de pequeño calibre de los sujetos con SBA, presenten signos indicativos de alteraciones ultramicroscópicas en relación a las de la población control, que nos podrían orientar hacia investigaciones que mejor puedan definir los signos de "sufrimiento" de la fibras nerviosas periféricas de pequeño calibre. Esto nos motiva a realizar una ulterior ampliación cuantitativa y cualitativa de la investigación visto el número reducido de biopsias hasta ahora realizadas.

\section{DISCUSIÓN Y CONCLUSIONES}

El dato de mayor relevancia, extrapolado desde las pruebas neurológicas realizadas en una población de sujetos no seleccionados afectados por el síndrome de la boca ardiente, es la elevada frecuencia de una disminución de la sensibilidad para estímulos térmicos en la lengua. Esta observación no está de acuerdo con los resultados del único estudio descrito en la literatura, sobre la sensibilidad de la mucosa lingual, de Gruschka y cols. que examinaron 70 pacientes con el síndrome de la boca ardiente (48). A las conclusiones alcanzadas con este trabajo nosotros reservamos dos críticas. En primer lugar la discordancia de los datos puede ser una consecuencia de los distintos instrumentos empleados, un estimulador térmico de gran superficie $\left(12,5 \mathrm{~cm}^{2}\right)$ en nuestro trabajo y un estimulador térmico de pequeña superficie $\left(49 \mathrm{~mm}^{2}\right)$ en el de Gruschka y cols. Para

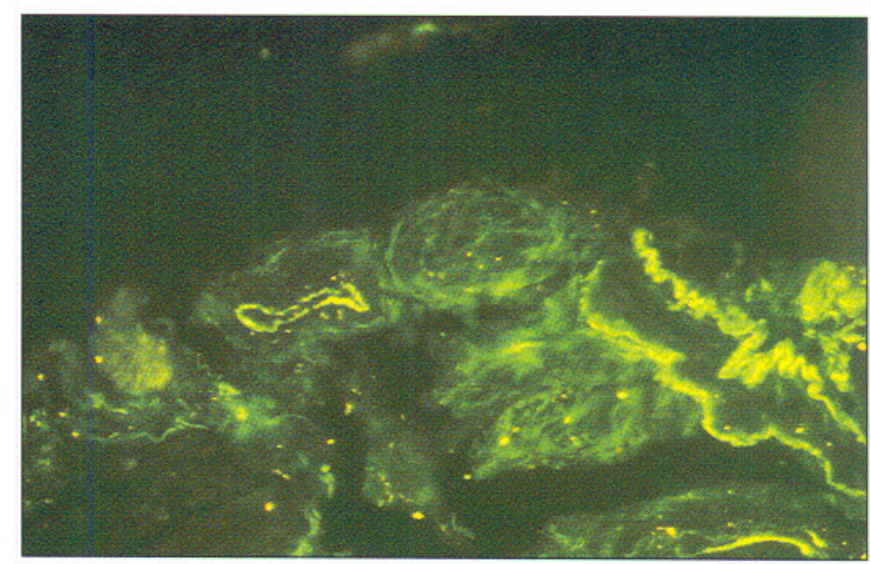

Fig. 2. Examen de la mucosa oral por medio de inmunofloresencia con el uso del anticuerpo protein gene product 9.5. (x 250). 
nosotros el aparato empleado por Gruschka y cols. es adecuado para la detección de las alteraciones de las sensibilidad térmica de las fibras nerviosas que transportan la sensibilidad desde campos receptores de grandes dimensiones, en algún caso sin deficit funcional. Además Gruschka y cols. concluyen mencionando la ausencia de alteraciones de la sensibilidad térmica de la lengua en pacientes con síndrome de la boca ardiente, sobre la base de una elaboración estadística, que relaciona el umbral de percepción de los estímulos térmicos en los pacientes afectados por el síndrome de la boca ardiente comparado con los datos obtenidos de una población control. En estos términos la alteración de la percepción a los estímulos térmicos, evidenciadas por nosotros, en algunas personas, sería más baja de la normal umbral de percepción de otros pacientes y no llevaría a una significación estadística. Sin embargo si se considera el número de observaciones de las alteraciones del umbral de percepción de los estímulos térmicos, y no el valor de la temperatura del umbral de percepción, y se relaciona estadísticamente el dato con lo obtenido desde una población control, la hipótesis de la ausencia de diversidad en las dos poblaciones seria rechazada. La alteración de la percepción de los estímulos térmicos, apreciada en los pacientes con el síndrome de la boca ardiente, no puede ser consecuencia de patologías primitivas de la mucosa lingual. Alteraciones macroscópicas y microscópicas de la mucosa lingual, como por ejemplo la presencia de queratosis, han sido excluidas del examen objetivo y del examen histológico de las biopsias. La disminución de la sensibilidad hacia los estímulos térmicos parece contrariamente ser la expresión de una alteración selectiva de las fibras nerviosas de pequeño calibre. Es bien conocido, que mientras la sensibilidad hacia los estímulos mecánicos es transportada por medio de las fibras nerviosas mielínicas de gran calibre, tipo A beta según la clasificación de Erlanger e Gasser, la sensibilidad para el dolor de calidad pinchante y para el frío, está transportada por fibras nerviosas mielinicas de pequeño calibre, de tipo A delta, y finalmente la sensibilidad al calor y de los estímulos dolorosos de cualidad ardiente está transportada sobre todo para las fibras nerviosas amielinicas, de tipo C. Varias noxae patógenas del nervio periférico afectan sobre todo al eje nervioso más que a la mielina, dañando así en primer lugar las fibras nerviosas amielinicas o poco mielinizadas, y provocando por lo tanto una selectiva disminución de la sensibilidad de los estímulos térmicos, y en fases más avanzadas de la enfermedad también una hipoalgesia. Entre los casos más frecuentes hay que mencionar la neuropatía diabética, mientras que son más raras las neuropatías por amiloidosis, por desequilibrio metabólico durante el curso de disfunciones de la glándula tiroides y algunas avitaminosis. En los sujetos que nosotros hemos examinados ha sido frecuentemente evidenciada una disminución de la sensibilidad para estímulos térmicos, que interesaban además de la lengua, también las extremidades de las extremidades inferiores, como típicamente se aprecia en las polineuropatías. Desde los datos extrapolados de la anamnésis y desde los exámenes realizados se ha además evidenciado que, un elevado porcentaje de sujetos examinados estaba afectado de alguna patología: la diabetes, la hepatitis viral del tipo B y/o C, hipovitaminosis B 12 o sideropenia, que notoriamente están en relación con la génesis de polineuropatías de tipo axonal. También otros autores han evidenciado una elevada frecuencia de estas enfermedades en los pacientes afectados por el síndrome de la boca ardiente, sin embargo estas enfermedades no han sido directamente relacionadas a la presencia de polineuropatías. Es importante evidenciar que, en muchos casos que nosotros hemos observado, las manifestaciones de la polineuropatía interesaban exclusivamente por las funciones transmitidas por las fibras nerviosas de pequeño calibre, sin afectar la funcionalidad motora de los reflejos óseo-tendón y con un normal examen electromiográfico, cuando se había realizado. Por esta razón mencionamos los resultados del estudio de las sensibilidades de la lengua de Gruscka y cols, que no han evidenciado alteraciones de las sensibilidades transportadas por medio de las fibras nerviosas mielínicas de gran calibre. La presencia de una disminución de la sensibilidad para los estímulos térmicos de la lengua, así como de las extremidades de las extremidades inferiores, es siempre expresión de una lesión grave de las fibras nerviosas, generada por un daño prolongado en el tiempo. En un primer momento, por la elevada capacidad de regeneración de las fibras nerviosas y por el número tan elevado de fibras nerviosas presentes en relación a la función que tienen que realizar, la función de transmisión de las señales de sensibilidad están mantenidas. En esta fase la gene- 
ración de terminales nerviosos extremadamente sensibles y el aumento de la densidad de receptores es responsable de una disminución del umbral y del estímulo necesario para provocar el dolor, hiperalgesia. Además la lesión nerviosa puede producir una respuesta refleja de liberación de componentes inflamatorios, en primer lugar la sustancia P, por parte de los terminales nerviosos sensitivos, provocando una inflamación neurogénica. Estos procesos son la base de manifestaciones dolorosas en el curso de una polineuropatía, como por ejemplo la neuropatía diabética hiperálgica. En estos casos el paciente refiere un dolor espontáneo de tipo ardiente, agravado para el contacto con superficies calientes, también de temperatura no elevada, y aliviado para el contacto con objetos fríos, con un aspecto de la piel enrojecido y edematoso. Las característica del dolor referido en las extremidades inferiores en estos sujetos son por lo tanto extremadamente parecidas con aquellas descritas de nuestro pacientes afectados por el síndrome de la boca ardiente, como resulta del análisis de los descriptores de la calidad del dolor del cuestionario del dolor de McGill. Es por lo tanto posible que la presencia de una disminución de la sensibilidad térmica de la lengua se pueda relacionar con una lesión terminal e irreversible de las fibras nerviosas y que el dolor ardiente sea contrariamente expresión de una alteración precoz, "iritativa " de las mismas fibras. Naturalmente un proceso neuropático afectaría en grado distinto a la población de fibras nerviosas: para algunas fibras nerviosas la lesión seria mínima, sin un déficit de la función sensitiva pero con fenómenos sensitivos positivos, como la hiperalgesia al calor y el dolor espontáneo ardiente; en otras fibras, en el mismo momento, la lesión podría ser más avanzada, con desaparición de las molestias sensitivas positivas pero con un evidente déficit de la percepción como el revelado en el examen cuantitativo de la sensibilidad. Las hipótesis de alteraciones neuropáticas, descritas en la literatura de forma exhaustiva en distintos síndromes dolorosos neuropáticos, explican adecuadamente las manifestaciones del síndrome de la boca ardiente y de los datos de los exámenes neurofisiológicos realizados en los pacientes examinados en nuestro trabajo. Recordamos que una alteración del umbral de percepción de un estimulo térmico en la lengua ha sido evidenciada solo en una minoría, pero relevante, de los pacientes afectados por el síndrome de la boca ardiente, y por lo tanto no representa una condición imprescindible para la génesis de el síndrome doloroso, pero más que nada un fenómeno asociado al sufrimiento de las fibras nerviosas, que es el común denominador de todos los fenómenos sensitivos deficitarios y positivos.

Los estudios epidemiológicos evidencian que, el síndrome de la boca ardiente es frecuentemente una molestia temporal limitada con una duración media de algunos años. Se puede pensar que la remisión del dolor no sea debida a una resolución de la neuropatía y de la causa neuropática, sino más bien un agravamiento de la lesión con una desaparición de los fenómenos sensitivos positivos.

Desgraciadamente no hemos realizados estudios neurofisiológico en pacientes que actualmente no presentan SBA, pero que la tuvieran en el pasado. $\mathrm{Ni}$ estudios parecidos se han descrito en la literatura, y no podemos por lo tanto confirmar esta hipótesis; por otro lado los síndromes dolorosos por polineuropatía presenta generalmente una evolución limitada en el tiempo, no relacionada o además inversamente relacionada a la gravedad de la enfermedad.

Si el síndrome de la boca ardiente es o no la expresión clínica de una polineuropatía de las fibras de pequeño calibre no está todavía muy claro porque se manifiesta en la cavidad oral, sin interesar en muchos casos las fibras nerviosas más largas que transportan la sensibilidad a las extremidades inferiores. Una explicación podría ser que la lengua es una localización corporal con mayor densidad de fibras nerviosas sensitivas, y que por lo tanto más fácilmente podrían desarrollarse en esta región lesiones nerviosas "irritatvas". La temperatura de la cavidad oral, y de la mucosa lingual en particular, como se ha demostrado con la tele-termografía que hemos realizado sobre nuestros pacientes, resulta significativamente elevada en comparación a los otras localizaciones corpóreas. En los síndromes dolorosos neuropáticos el contacto con objetos de temperaturas superiores a los $36^{\circ} \mathrm{C}$, que se alcanzan regularmente y adelantadamente en la cavidad oral, pueden provocar un dolor quemante. Esta condición puede manifestarse, además porque como en la cavidad oral, también en otras localizaciones corporales, como la mucosa de la vulva, que puede ser sede de 
dolor de análoga calidad que la del síndrome de la boca ardiente. Los sujetos afectados por el síndrome de la boca ardiente frecuentemente refieren un alivio del dolor al contacto con alimentos fríos que de forma rápida disminuyen la temperatura de la mucosa. En condiciones de hiperalgesia por neuropatía hay una susceptibilidad aumentada de las terminaciones nerviosas libres, apta para la recepción de los estímulos dolorosos, hacia estímulos químicos, condición que no suele verificarse en el cutis pero que frecuentemente se presenta en la mucosa oral. Por esto la ingesta de alimentos ácidos, como el vinagre o el zumo de limón, pueden acentuar el dolor en los sujetos afectados por el síndrome de la boca ardiente. Se menciona la aplicación de ácido acético por parte de los ginecólogos, como prueba para diferenciar la vulvo-dinia esencial de otros dolores vulgares de otra naturaleza (por ejemplo el psicógeno), sobre la mucosa de la vulva como agravante del dolor quemante. Las consideraciones hechas no pueden explicar la elevada prevalencia de las mujeres en edad post-menopausia en los pacientes afectados por el síndrome de la boca ardiente. Es posible que exista una relación entre la alteración de los valores hormonales femeninos y la distrofia de las mucosas consecuente a la aparición de las manifestaciones neuropáticas a cargo de las mucosas (incluyendo también la vulvodinia). Pero en nuestros pacientes, así como mencionado en la literatura, no se ha evidenciado una atrofia de las mucosas linguales, y por el otro lado una terapia estrogénica no parece modificar las molestias del síndrome de la boca ardiente. Es por lo tanto evidente que parte del proceso patológico del síndrome de la boca ardiente todavía se aleja de cualquier posibilidad de análisis.

\section{BIBLIOGRAFÍA}

1. Rhodus NL, Myers S, Bowles W, Schwartz B, Parsons $\mathrm{H}$. Burning mouth syndrome: diagnosis and treatment. Northwest Dent. 2000 May-Jun; 79 (3): 21-8.

2. Muzyka BC. Diagnosis and treatment of burning mouth syndrome. Pract Periodontics Aesthet Dent. 2000 Aug; 12 (6): 568.
3. Muzyka BC, De Rossi SS. A review of burning mouth syndrome. Cutis. 1999 Jul; 64 (1): 29-35. Review.

4. Miyamoto SA, Ziccardi VB. Burning mouth syndrome. Mt Sinai J Med. 1998 Oct-Nov; 65 (5-6): 343-7. Review.

5. Lauritano D, Spadari F, Formaglio F, Zambellini Artini M, Salvato A. [Etiopathogenic, clinicaldiagnostic and therapeutic aspects of the burning mouth syndrome. Research and treatment protocols in a group of patients]. Minerva Stomatol. 1998 Jun; 47 (6): 239-51. Review. Italian.

6. Vescovi P, Manfredi M, Savi A, Fazzi M, Bonanini M. [Burning mouth syndrome. Clinical experience with 75 patients]. Minerva Stomatol. 2000 Apr; 49 (4): 169-77. Review. Italian.

7. Main DMG, Basker RM. Patients complaning of mouth: further experience in clinical assessment and management. Br Dent J 1983; 154: 206-1 1.

8. Gorsky M. Burning mouth: a rewiev and update. Ontario Dentist. 1983; 60: 56-61.

9. Gorsky M, Silverman S Jr, Chinn H. Clinical characteristics and management outcome in the burning mouth syndrome: an open study of 130 patients. Oral Surg Oral Med Oral Pathol. 1991; 72: 192-5.

10. Domb GR Chole RA. The burning mouth and tongue. Ear Nose Throat. J 1981; 60: 29, 31, 346.

11. Gorsky M, Silverman S Jr, Chinn H. Burning mouth syndrome : a review of 98 cases. J Oral Med 1987; 42: 79.

12. Zagarelli DJ. Burning mouth: analysis of 57 patients. Oral Med Oral Surg Oral Pathol 1984; 58: 34-8.

13. Grushka M, SessleBJ. Burning mouth syndrome. Dent Clin North Am. 1991; 35: 171-84. 
14. Van der Waal I. The burning mouth syndrome. Copenhagen. Munsgaard, 1990.

15. Formaker BK, Frank ME. Taste function in patients with oral burning. Chem Senses. 2000 Oct; 25 (5): 575-81.

16. Jensen JL, Barkvoll P. Clinical implications of the dry mouth. Oral mucosal diseases. Ann N Y Acad Sci. 1998 Apr 15; 842: 156-62. Review.

17. Mott AE, Grushka M, Sessle BJ. Diagnosis and managements of taste disorders and burning mouth syndrome. Dent Clin North Am. 1993; 37: 33-71.

18. Carlson CR, Miller CS, Reid KI. Psychosocial profiles of patients with burning mouth syndrome. J Orofac Pain. 2000 Winter; 14 (1): 59-64.

19. Nicholson M, Wilkinson G, Field E, Longman L, Fitzgerald B. A pilot study: stability of psychiatric diagnoses over 6 months in burning mouth syndrome. J Psychosom Res. 2000 Jul; 49 (1): 1-2.

20. Pokupec-Gruden JS, Cekic-Arambasin A, Gruden V. Psychogenic factors in the aetiology of stomatopyrosis. Coll Antropol. 2000 Jul; 24 Suppl 1: 119-26.

21. Bogetto F, Maina G, Ferro G, Carbone M, Gandolfo S. Psychiatric comorbidity in patients with burning mouth syndrome. Psychosom Med. 1998 May-Jun; 60 (3): 378-85.

22. Trikkas G. Further psychiatric considerations of glossodynia. Psychosomatics. 1998 Jan-Feb; 39 (1): 85-7.

23. Van der Ploeg W, van der Waal N, Eijkman MAJ. Psychological aspects of patients with burning mouth syndrome. Oral Surg Oral Med Oral Pathol 1987; 63: 664-8.

24. Browning S, Hislop S, Scully C. The association between burning mouth syndrome and psychosocial disordes. Oral Surg Oral Med Oral Pathol. 1987; 64: 171-4.
25. Eli I, Kleinhauz M, Baht R. Antecedents of burning mouth syndrome (glossodynia): recent life events vs. psychopathologic aspects. J Dent. 1989; 73: 567-72.

26. Hakeberg M, Berggren U, Hagglin C, Ahlqwist M. Reparated burning mouth symptoms among middle-aged and elderly women. Eur J Oral Sci. 1997 Dec; 105 (6): 539-43.

27. Ferguson MM, Carter J, Boyle P. Oral complaints related to climacteric symptoms in oophorectomized women . J R Soc Med. 1981; 74: 492-8.

28. Lamey PJ, Lamb AB. The usefulness of the HAD scale in assessing anxiety depression in patientes with disordes. Oral mouth syndrome. Oral Surg Oral Med Oral Pathol. 1989; 67: 390-2.

29. Koblenzer CS, Bostrom P. Chronic cutaneuos dysesthesia syndrome: A psychotic phenomenon or a depressivive symptom? J Am Acad Dermatol. 1994; 30: 370.

30. Heckmann SM, Heckmann JG, Hilz MJ, Popp M, Marthol H, Neundorfer B, Hummel T. Oral mucosal blood flow in patients with burning mouth syndrome. Pain. 2001 Feb 15; 90 (3): 281-6.

31. Lamey PJ, Murray BM, Eddie SA, Freeman RE. The secretion of parotid saliva as stimulated by $10 \%$ citric acid is not related to precipitating factors in burning mouth syndrome. J Oral Pathol Med. 2001 Feb; 30 (2): 121-4.

32. Lundy FT, Al-Hashimi I, Rees TD, Lamey PJ. Evaluation of major parotid glycoproteins in patients with burning mouth syndrome. Oral Surg Oral Med Oral Pathol Oral Radiol Endod. 1997 Feb; 83 (2): 252-8.

33. Navazesh $M$. Salivary gland hypofunction in elderly patients. J Calif Dent Assoc. 1994; 22: 62-8.

34. Brody HA, Prendergast JL, Silverman S Jr. The relationship between orall symptoms, insulin release, and glucose intolerance. Oral Surg Oral Med Oral Pathol. 1971; 31: 777-82. 
35. Samaranayake LP, Lamb AB, Lamey O-J. Oral carriage of Candida species and coliforms in patients with burning mouth syndrome. J Oral Pathol Med. 1989; 18: 233-5.

36. Kleinegger CL, Krolls SO. Severe pernicious anemia presenting with burning mouth symptoms. Miss Dent Assoc J. 1996 Spring; 52 (1): 12-4. Review

37. Lamey PJ, Hammond A, Allam BF. Vitamin status of patients with burning mouth syndrome and the response to replacement therapy. $\mathrm{Br}$ Dent J. 1986; 160: 81-3.

38. Lamey PJ. Burning mouth syndrome: approach to successful management. Dent Update. 1998 Sep; 25 (7): 298-300.

39. Woda A, Navez ML, Picard P, Gremeau C, Pichard-Leandri E. A possible therapeutic solution for stomatodynia (burning mouth syndrome). J Orofac Pain. 1998 Fall; 12 (4): 272-8.

40. Marbach JJ. Medically unexplained chronic orofacial pain. Temparaomandibular pain and dysfunction syndrome, orofacial phantom pain, burning mouth syndrome, and trigeminal neuralgia. Med Clin North Am. 1999 May; 83 (3): 691-710. Review.

41. Verdugo R, Ochoa JL. Quantitative somatosensory thermotest - a key method for functional evaluation of small calibre afferent channels. Brain. 115: 893-913, 1992.

42. Gruener G, Dyck PJ. Quantitative sensory testing: methodology, applications and future dírections. J Clin Neurophysiol. 1994; 11: 568-583.
43. Cline MA, Ochoa J, Torebjork HE. Chronic hyperalgesia and skin warming caused by sensitizcd $C$ nociceptors. Brain. 1989; 112: 621-647.

44. Lacerenza M., Marchettini P., Formaglio F., Marangoni C. e Smirne S. Neurophatic pain: defínition, up dated clinical approach and instrumental evaluation. Funct Neurol. 1993; 8: 57-64.

45. Dalsgaard CJ, Rydl M. e Haegerstrand A. Cutaneous innervation in man visualized with protein gene product 9.5 (PGP 9.5) quantibodies. Hystochemistry. 1989; 92: 385-9.

46. Gubelkian S.,Wharton J. e Polak JM.The visualization of cardiovascular innervation in the Guinea pig using an antiserum to protein gene product 9.5 (PGP 9.5). J Auton Nerv Syst. 18: 235 $47,1987$.

47. Grushka M., Sessle BJ and Miller R. Pain and personality prolfiles in burning mouth syndrome. Pain. 1987; 28: 155-167.

48. Grushka M., Sessle BJ and Howley TP. Psycophisycal assesment of tactile and termal sensory function in burning mouth syndrome. Pain. 1987; 28: 159-184.

\section{CORRESPONDENCIA}

Dott. Dorina Lauritano

via Clara Maffei $n^{\circ} 3$

24121 Bergamo

Tel: 035574838

e-mail: lauritano@qui.it 